Übermittlung sensibler Daten über das WWW - etwa beim Onlinebanking - für Anbieter entscheiden, die mehrstufige Authentifizierungsverfahren bei der Abwicklung von Transkationen anbieten.

Weitere Informationen finden sich unter www.mirapoint.com.

Helmut Reimer

\section{Buch- besprechungen}

Gola, Peter; Jaspers, Andreas: Das BDSG im Überblick, Erläuterungen, Schaubilder und Organisationshilfen zum BDSG für die Datenschutzpraxis, DATAKONTEXT Fachverlag, Frechen, 3. überarbeitete und erweiterte Auflage 2006, 88 Seiten (DIN A4), ISBN 3-89577-376-X, 24,- Euro

Beim Bundesdatenschutzgesetz (BDSG) handelt es sich um eine äußerst komplexe Regelungsmaterie, die sich über den gesetzlichen Wortlaut nur erschließen lässt. Ursächlich hierfür ist eine zweimalige Novellierung, ohne dabei jedoch die Gesamtkonzeption zu ändern oder die Verständlichkeit zu erhöhen. Die vorliegende dritte Fassung des BDSG enthält neben den aus den EUVorgaben zwingend resultierenden nationalen Gesetzesänderungen bereits einige Grundsätze „modernen Datenschutzrechts“, wie die dem sog. Systemdatenschutz zuzuordnenden Prinzipien der Datenvermeidung und Datensparsamkeit ( $\S 3$ a BDSG) oder Möglichkeiten des Datenschutzaudits ( $\$ 9 \mathrm{a}$ BDSG), ebenso erste Ansätze zu einer Selbstregulierung des Datenschutzes durch die Anwender (über allgemeine Standesgrundsätze oder unternehmensinterne „Binding Corporate Rules - BCR"), schließlich auch Regelungen zur Videoüberwachung und $\mathrm{zu}$ den sog. mobilen Speicher- und Verarbeitungsmedien bzw. Chipkarten ( $\S \S 6$ $\mathrm{b}$ und $6 \mathrm{c}$ BDSG).

Bei der erforderlichen Umsetzung des Bundesdatenschutzgesetzes durch die Unternehmen und deren Datenschutzverantwortliche bietet die Arbeitshilfe von Gola/Jaspers insofern gute Hilfestellung als sie durch verständliche Erläuterungen und Schaubilder einen Einstieg in das Verständnis des Datenschutzrechts geben soll. Die Regelungen des BDSG sind in Themengebiete untergliedert und werden in Sachzu- sammenhängen systematisch erläutert. In der dritten Auflage dieses Praxisleitfadens wurden die Erläuterungen zu den einzelnen Kapiteln erweitert und an die aktuelle Interpretation des Gesetzes durch die Rechtsprechung und Aufsichtsbehörden angepasst. Die Schaubilder wurden ergänzt und erweitert. Weiterhin wurden Muster von Praxishilfen in das Werk integriert. Besonders hervorzuheben sind das in das Werk aufgenommene Muster einer betrieblichen Datenschutzrichtlinie (Organisationsrichtlinie/Arbeitsanweisung), ein Mitarbeiter-Merkblatt zum Datenschutz sowie eine Checkliste zur Umsetzung der 8 Gebote zur Datensicherheit (gem. der Anlage zu § 9 BDSG): Zutrittskontrolle, Zugangs-, Zugriffs-, Weitergabe-, Eingabe-, Auftrags-, Verfügbarkeitskontrolle sowie Gebot der Datentrennung. Am Ende der Publikation findet sich das BDSG in der zuletzt durch Gesetz vom 5.9.2005 geänderten Fassung vollständig abgedruckt.

Die Verfasser verfügen über profunde Kenntnisse und langjährige Erfahrungen im Bereich des Datenschutzes. Gola ist als Vorsitzender und Jaspers als Geschäftsführer der Gesellschaft für Datenschutz und Datensicherung e.V. (GDD) u.a. mit der Unterstuitzung und Beratung der Datenschutzbeauftragten und -verantwortlichen von Unternehmen und Behörden betraut, zudem sind beide Autoren im Rahmen der GDD-Datenschutz-Akademie als Referenten zur Vermittlung der Basisqualifikation für betriebliche Datenschutzbeauftragte tätig.

Die Publikation wendet sich über letztgenannten Adressatenkreis hinaus aber auch besonders an die Datenschutzverantwortlichen in den Fachabteilungen von Unternehmen und Behörden sowie an Betriebs/Personalratsmitglieder. Die systematische Darstellung des BDSG anhand von Schaubildern ermöglicht es den Datenschutzverantwortlichen, die komplexe Materie des BDSG sowohl sich selber zu erschließen als auch den mit der Verarbeitung personenbezogener Daten betrauten Mitarbeitern zu vermitteln. Insoweit empfiehlt sich die Darstellung auch durchaus zur gesetzlich gebotenen Mitarbeiterschulung in Fragen von Datenschutz und Datensicherheit.

Dr. Thomas P. Stähler
Spindler, Gerald (Hrsg.): Vertragsrecht der Internet-Provider, 2. Aufl. 2004, Verlag Dr. Otto Schmidt, Köln , 1024 Seiten, Preis 98,80€

Kaum ein Unternehmen ist heute nicht mehr mit Fragen befasst, die sich aus den Entwicklungen der New Economy ergeben. Das wirtschaftliche Wachstum im Bereich Online-Vertrieb von Waren und Dienstleistungen entwickelt sich stetig, was u.a. dazu geführt hat, dass der Vertriebskanal Internet für viele, auch mittelständische Unternehmen, sich zu einem wichtigen wirtschaftlichen Standbein entwickelt hat. Die Fragen die sich dabei in Bezug auf die Nutzung moderner Kommunikationswege nahezu täglich ergeben, sind, mit Ausnahme klassisch EDV-rechtlicher Fragestellungen, Gegenstand des von Spindler herausgegebenen Handbuches. Datenschutzrechtliche Erläuterung werden z.T. in den jeweiligen Kapiteln angesprochen. Es geht um Telekommunikationsleistungen, Website-Hosting, Domain-Recht, Webdesign, Hyperlinks, u.a.m. Mit der zweiten Auflage wurde das Werk aktualisiert und um die Themen Application Service Providing sowie Auktionsund Plattformverträge erweitert. Das Werk zeichnet sich durch die Tatsache aus, dass die bearbeiteten Themen nicht nur juristisch gründlich aufgearbeitet werden, sondern jeweils für die betreffende Fragestellung Gestaltungsvorschläge erarbeitet werden. Der Herausgeber hat sich damit bewusst gegen die Bereitstellung komplexe Musterverträge oder -Bedingungen entschieden. Darin kann man ihm nur beipflichten. Aufgrund der Komplexität aktueller Vertragsgestaltungsaufgaben kommen die Vertragspartner, so sie gut beraten sind, ohnehin nicht mehr um die individuelle Gestaltungen einer Vertragsbeziehung umhin. Hier gibt das Werk wichtige Hinweise und erzeugt das notwendige Maß an Problembewusstsein. Damit bietet das Werk nicht nur wichtige Informationen für einen ersten Einstieg in die bearbeiteten Themenfelder, sondern stellt darüber hinaus ein exzellentes Werkzeug dar, das im Rahmen der Vorbereitung und Durchführung von Vertragsgestaltung- und Verhandlung zur Nutzung uneingeschränkt empfohlen werden kann.

\section{A. Müglich}

